\title{
ROBOT SALAMANDRA ANFIBIO CON LOCOMOCIÓN BIOINSPIRADA
}

\section{ROBOTICS AMPHIBIOUS SALAMANDER WITH BIO-INSPIRED LOCOMOTION}

\author{
Marco A. Luna ${ }^{1}$, Julio F. Moya ${ }^{1}$, Wilbert G. Aguilar ${ }^{2,3,4, *}$, Vanessa Abad $^{5}$
}

\section{Resumen}

En este artículo se presenta el desarrollo de un robot anfibio con una dinámica de movimiento bioinspirada en la locomoción de la salamandra (Cryptobranchidae). El robot es teleoperado mediante una aplicación para dispositivos móviles (Smartphones, tablets, etc.). Se propone una estructura que permita al robot llevar a cabo dos acciones: caminar y nadar. Los movimientos de una salamandra real se han estimado basándose en una cámara cenital y se ha diseñado un algoritmo de control de locomoción que replique esos movimientos. El desempeño del robot se ha evaluado utilizando como métrica el error cuadrático medio entre el movimiento del robot y de la salamandra obteniendo errores menores al 5 $\%$ en los ángulos de movimiento de la espina dorsal.

Palabras clave: cinemática, locomoción, robótica, robots anfibios, salamandra.

\begin{abstract}
This paper presents the development of an amphibious robot with a motion dynamics bioinspired on the locomotion of the salamander (Cryptobranchidae). The robot is teleoperated by an application for handled devices. We propose a structure to perform two different motions: walk and swim. We extract the movements from a real salamander by a zenith camera, and a locomotion control algorithm is designed to reply this movements. We evaluate the performance of the robot in comparison with the real animal movements using the RMSE (Root Mean Square Error) as metric of evaluation. We obtain errors less than $5 \%$ in the angles of backbone movement.
\end{abstract}

Keywords: Amphibious Robot, Kinematics, Locomotion, Robotics, Salamander.

\footnotetext{
${ }^{1}$ Departamento de Eléctrica y Electrónica DEEE, Universidad de las Fuerzas Armadas ESPE, Sangolquí - Ecuador.

${ }^{2}$ Departamento de Seguridad y Defensa DESD, Universidad de las Fuerzas Armadas ESPE, Sangolquí - Ecuador.

${ }^{3}$ Centro de Investigación Científica y Tecnológica del Ejército CICTE, Universidad de las Fuerzas Armadas ESPE, Sangolquí - Ecuador.

${ }^{4, *}$ Grup de Recerca en Enginyeria del Coneixement GREC, Universitat Politècnica de Catalunya UPC, Barcelona - España. Autor para correspondencia w waguilar@espe.edu.ec

${ }^{5}$ Departament de Genètica, Universitat de Barcelona UB, Barcelona - España.
}

Recibido: 01-11-2016, aprobado tras revisión: 20-12-2016

Forma sugerida de citación: Luna, M.; Moya, J.; Aguilar, W.; Abad, V. (2017). «Robot salamandra anfibio con locomoción bioinspirada». INGENIUS. N. ${ }^{\circ} 17$, (Enero-Junio). pp. 51-59. ISSN: 1390-650X. 


\section{Introducción}

La salamandra es un anfibio cercano a los primeros tetrápodos [1], cuya dinámica de movimiento requiere un gasto energético bajo respecto a las dimensiones de su cuerpo, a diferencia de los robots terrestres de estructura cuadrúpeda $[2,3]$ o aéreos de estructura cuatirotor $[4,5]$. Cuando la salamandra se desplaza en tierra, la forma de movimiento que utiliza es la reptación, es capaz de moverse utilizando pequeñas extremidades. La dinámica de movimiento de la salamandra es óptima desde el punto de vista de consumo energético y adaptable a diversos entornos [6,7], por lo que algunos de los robots bioinspirados utilizan esta estructura.

Cuando la salamandra se desplaza en tierra el movimiento lo lleva a cabo coordinando las extremidades en forma de $\mathrm{X}$ (se mueven la pata superior derecha junto con la inferior izquierda y viceversa). Adicionalmente, la espina dorsal lleva a cabo un movimiento oscilatorio que contribuye al movimiento general de la salamandra. En agua, las extremidades son orientadas hacia la parte trasera permitiendo que la mayor parte del trabajo lo realice la espina dorsal [4]. Las patas de la salamandra gigante son pequeñas respecto a su cuerpo y no contribuyen significativamente a la locomoción del animal.

Estudios realizados en [9] y [8] muestran que la locomoción de la salamandra depende en mayor medida de la espina dorsal. En animales con extremidades pequeñas, la flexión lateral de la columna contribuye al movimiento en entornos acuáticos y facilita el movimiento en entornos terrestres [1]. En [10] se analiza la cinemática de la salamandra para usarla como base de algoritmos de locomoción en robots. En ese trabajo los autores utilizan técnicas de seguimiento de movimientos y calibración de cámaras de video.

En la literatura se puede encontrar un amplio número de robots inspirados en morfología animal como: serpientes [11-14], peces [15], langostas [16], hexápodos [17], entre otros. Algunos robots, presentados en [18] y [19], no tienen estructuras bioinspiradas, no obstante, se pueden desplazar en ambientes acuáticos y terrestres.

Existen pocos prototipos de robots salamandra como el descrito en [1] que cuenta con dos grados de libertad (DOF) para la columna vertebral, y dos para cada extremidad. Sin embargo, la movilidad de este robot es limitada y está enfocado en estudios biológicos. El Pleurobot presentado en [20] es un robot autónomo terrestre con cuatro grados de libertad en cada pata y utilizado en aplicaciones de rescate. En [21] se presenta un robot salamandra autónomo, capaz de caminar y nadar, con diseño hidrodinámico y modular. Algunos robots con patas y columna vertebral flexible son construidos por aficionados, con descripciones en Internet, no orientados a propósitos científicos.
Nuestro enfoque propone la utilización de materiales de bajo costo para la construcción de un robot teleoperado, con estructura modular hidrodinámica, utilizando un número de articulaciones reducido para simular el movimiento de la salamandra y desplazarse en entornos acuáticos y terrestres.

El resto del artículo está organizado de la siguiente forma: En la siguiente sección, se presenta una descripción del diseño de nuestro prototipo propuesto. Los resultados y discusión son presentados en la sección 3 y, finalmente, la sección 4 está destinada a conclusiones y trabajos futuros.

\section{Diseño del prototipo}

\subsection{Diseño mecánico}

El sistema debe ser capaz de desplazarse en entornos acuáticos y terrestres, es decir, que debe ser impermeable. Para garantizar la impermeabilidad del sistema se llevaron a cabo pruebas en las que se sumergió en agua cada uno de los elementos, durante una hora, a una profundidad de un metro y se comprobó que no existiesen filtraciones. Otra de las características que el robot debe poseer es la flotabilidad. El esqueleto se ha construido con polietileno, un elemento con una densidad menor a la del agua $\left(0,905 \mathrm{~g} / \mathrm{cm}^{3}\right)$.

Cada una de las secciones del cuerpo de la salamandra ha sido diseñada en un software CAD (Computer Assistance Design) y se describen a continuación:

\section{1) Espina dorsal}

La espina dorsal está constituida por cuatro módulos con el objetivo de alcanzar una flexibilidad comparable con la de la salamandra. Cada módulo contiene en su interior elementos mecánicos de transmisión de movimiento y componentes electrónicos que serán explicados en la sección 2.2. Los módulos se han diseñado con base en estructuras previas propuestas en [11], [22], [10], en las que cada módulo posee un DOF y está conformado por cuatro partes estructurales: cuerpo, tapa y dos conectores. (Figura 1). Los componentes mecánicos como electrónicos deben ser distribuidos en el interior de los módulos optimizando el espacio.

El diseño modular permite que las piezas puedan ser montadas y desmontadas de forma rápida. Los daños en un componente no comprometerán a todo el sistema. En la Figura 1 se puede observar que los módulos no son cúbicos sino poliedros lo que mejora su desempeño hidrodinámico. Los dos motores encargados del movimiento de la columna se encuentran acoplados a una transmisión tipo cadena en el interior de cada módulo. Estos motores son los responsables del movimiento de la espina dorsal como se muestra en la Figura 2. 


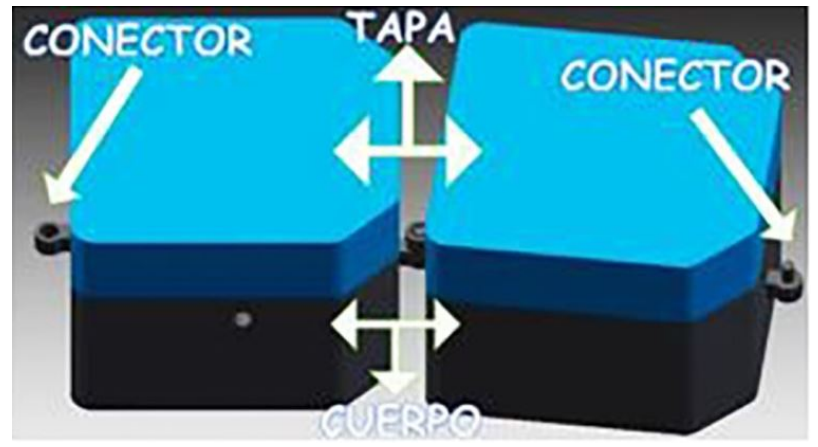

Figura 1. Partes estructurales del cuerpo. Los conectores tipo acople permiten la transmisión de movimiento entre módulos y la tapa brinda facilidad para montar y desmontar el circuito electrónico y los elementos mecánicos.

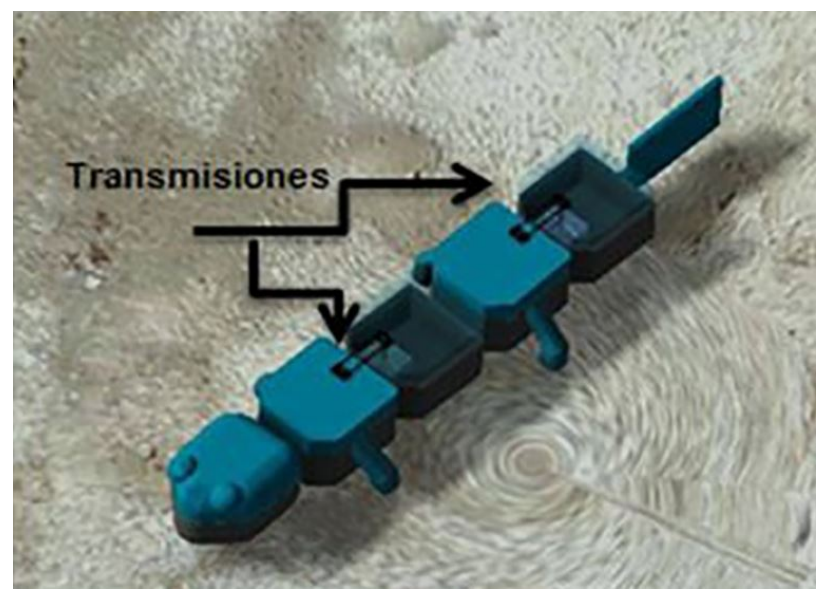

Figura 2. Elementos de transmisión de movimiento en el prototipo. Cada transmisión está acoplada a un motor para el movimiento.

\section{2) Extremidades}

Las extremidades poseen un DOF y están unidas a los elementos del cuerpo a través de un eje. A diferencia del animal real, tienen la capacidad de rotar 360 grados, sin embargo, su función es similar. En la Figura 3 se puede observar el diseño 3D de las extremidades.

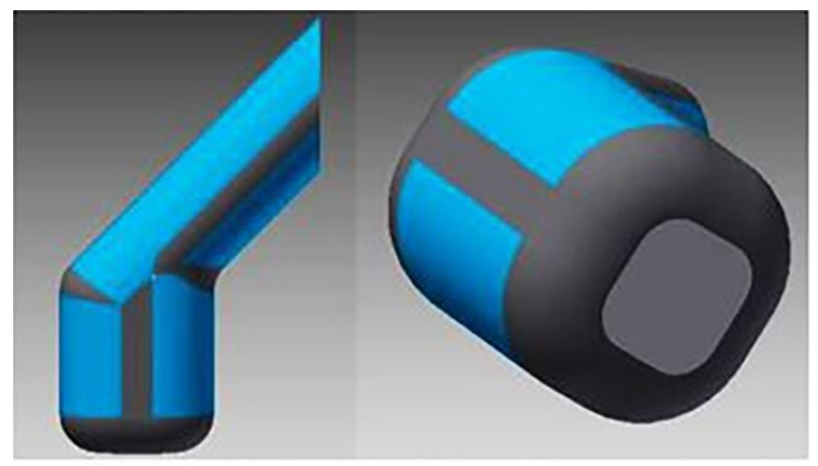

a)

b)

Figura 3. Extremidades. a) Vista frontal de las extremidades. b) Vista inferior del diseño 3D.
Durante la natación, las extremidades giran hacia atrás hasta alinearse con el cuerpo lo que permite al robot desplazarse con menor resistencia hidrodinámica. En la parte inferior poseen una superficie plana que maximiza el contacto con el suelo y la fricción necesaria para la tracción al caminar (Figura 3).

El diseño completo del prototipo se presenta en la Figura 4.

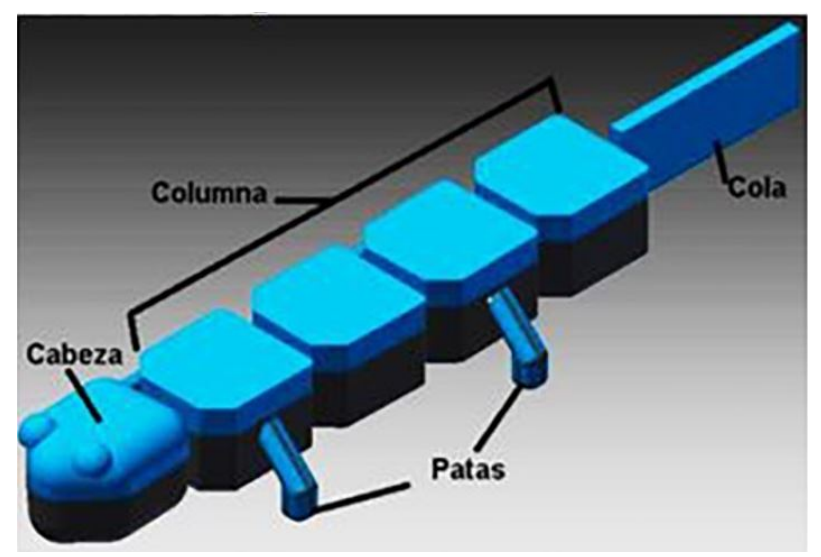

Figura 4. Cuerpo completo del robot salamandra. La cabeza y la cola son elementos que replican la morfología de la salamandra real, sin embargo, estos elementos no contribuyen a la dinámica de movimiento del robot.

\subsection{Diseño electrónico e interfaz}

El circuito del robot se alimenta de forma centralizada con una batería de litio para cuatro servomotores, un microcontrolador, dos encoders rotativos y un módulo bluetooth. La distribución de los elementos electrónicos se presenta en la Figura 5.

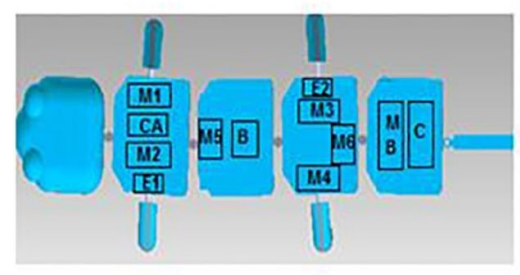

\begin{tabular}{|c|c|}
\hline Símbolo & Descripción \\
\hline$M 1$ & Motor 1 \\
\hline $1 / 2$ & Motor 2 \\
\hline$M 3$ & Motor 3 \\
\hline $1 M 4$ & Motor 4 \\
\hline MS & Motor 5 \\
\hline 106 & Notor 6 \\
\hline E1 & Encoder rotativo 1 \\
\hline E2 & Encoder rotativo 2 \\
\hline$C A$ & Creuto Acoplador \\
\hline 8 & Bsteria \\
\hline us & Modulo Bluetooth \\
\hline c & Controludor \\
\hline
\end{tabular}

Figura 5. Distribución de elementos electrónicos en el cuerpo del robot.

Se implantó un circuito de acondicionamiento para regular el voltaje de la batería de litio de 9 a $5 \mathrm{~V}$ usado en la alimentación del microcontrolador y el módulo bluetooth. El microcontrolador posee una interfaz serial para la transmisión y recepción de datos a través de una aplicación en Android.

Los servomotores de las patas se han acondicionado para la obtención del giro de $360^{\circ}$. La posición y el giro 
se controlan mediante PWM (modulación por ancho de pulso), con base en los datos obtenidos de un encoder rotativo electromecánico acoplado a una transmisión circular.

Para la selección del tipo de transmisión se realizaron pruebas utilizando engranes y poleas, cuyos resultados pueden ser observados en la sección 5 .

En el movimiento oscilatorio y la posición de la columna se utilizan dos servomotores truncados controlados por una segunda señal PWM.

Se desarrolló una aplicación para dispositivos inteligentes que permite controlar al robot mediante comunicación bluetooth. La interfaz humano-máquina (HMI) desarrollada para teleoperación se presenta en la Figura 6.

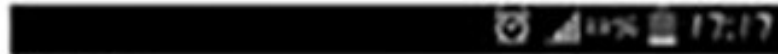 \\ Bareses \\ Sala mandra Bio-Inspirada}

\section{Conectar Bluetooth}

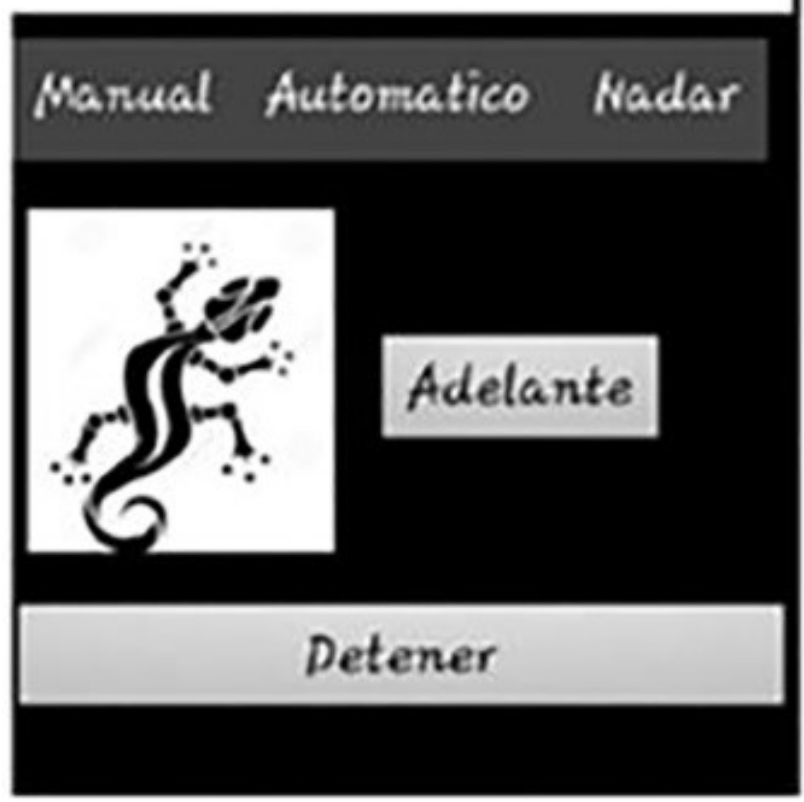

Figura 6. Interfaz de teleoperación del robot. Antes de iniciar es necesario que el usuario se conecte al robot por medio de bluetooth.

La interfaz de teleoperación recibe los comandos de alto nivel y ejecuta las acciones de control con base en los algoritmos de movimiento. El prototipo implementado se puede observar en la Figura 7.

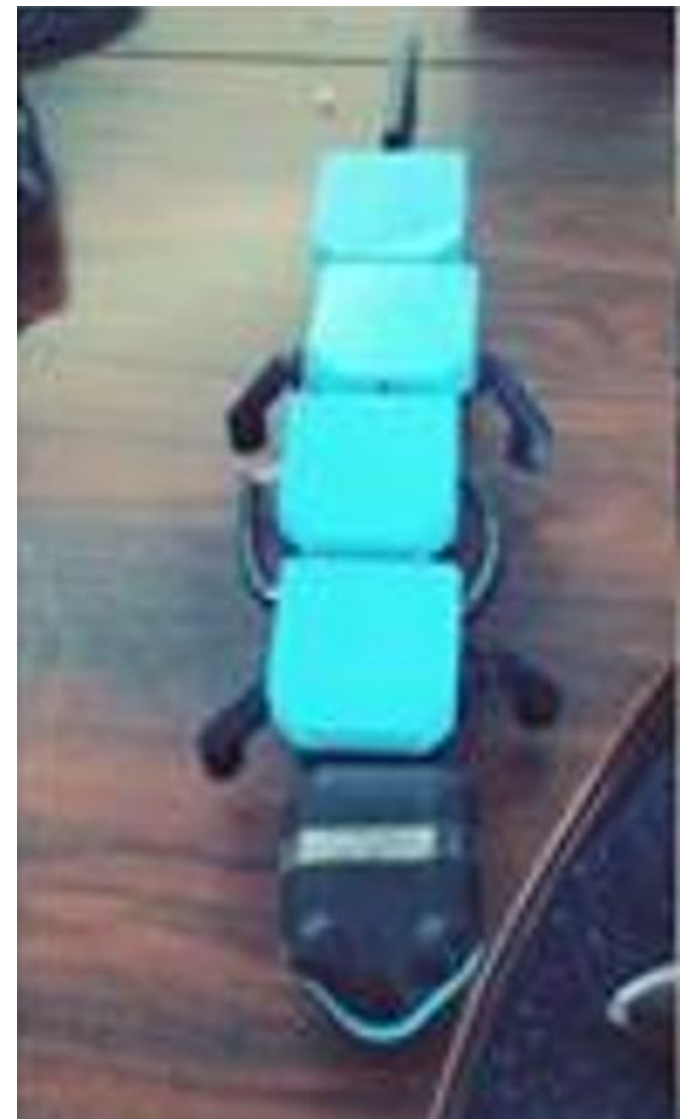

Figura 7. Prototipo implementado del robot salamandra.

\subsection{Algoritmos de movimiento}

Con base en el movimiento observado en el animal, se han diseñado dos algoritmos de locomoción (uno para agua y uno para tierra), los mismos que se presentan a continuación:

\begin{tabular}{l} 
Algoritmo $\begin{array}{c}\text { 1 Control de locomoción } \\
\text { en tierra }\end{array}$ \\
\hline Input: Posición Encoder 1 \\
Input: Posición Encoder 2 \\
1: if Posición Encoder $1=360$ grados then \\
2: Detener M1, M4 \\
3: Reiniciar Posición Encoder 2 \\
4: else \\
5: Mover M1, M4 \\
6: Giro M5 30 grados \\
7: Giro M6 -30 grados \\
8: end if \\
9: if Posición Encoder $2=360$ grados then \\
10: Detener M2, M3 \\
11: Reiniciar Posición Encoder 2 \\
12: else \\
13: Mover M2, M3 \\
14: Giro M5 -30 grados \\
15: Giro M6 30 grados \\
16: end if \\
\hline
\end{tabular}




\begin{tabular}{l}
$\begin{array}{c}\text { Algoritmo } 2 \text { Control de locomoción } \\
\text { en agua }\end{array}$ \\
\hline Input: Posición Encoder 1 \\
Input: Posición Encoder 2 \\
1: Mover M1, M4 \\
2: Mover M2, M3 \\
3: if Posición Encoder $1=90$ grados then \\
4: Detener M1, M4 \\
5: end if \\
6: if Posición Encoder $2=90$ grados then \\
7: Detener M2, M3 \\
8: end if \\
9: Giro M5 30 grados \\
10: Giro M6 -30 grados \\
11: Retraso 0,5 segundos \\
12: Giro M5 30 grados \\
13: Giro M6 -30 grados \\
14: to 9
\end{tabular}

En el algoritmo 1, el movimiento de las patas se lleva a cabo de forma simultánea con la espina dorsal lo que magnifica el movimiento.

En el algoritmo 2, la oscilación de la espina dorsal es más rápida, las patas se ubican hacia atrás en posición de nado permitiendo al robot desplazarse con su columna vertebral.

\subsection{Comparación con otras propuestas}

En la Tabla 1 se muestra una comparación de nuestro enfoque con prototipos propuestos en la literatura. Se analizan parámetros de diseño, debido a que en la literatura no se encontraron parámetros estandarizados de eficiencia.

Tabla 1. Comparación del primer prototipo con robots presentados en la literatura

\begin{tabular}{|c|c|c|c|}
\hline Robot & Columna vertebral & Extremidades & $\begin{array}{l}\text { Número de } \\
\text { motores }\end{array}$ \\
\hline Robo-Salamander [1] & $\begin{array}{l}\text { Posee } 2 \text { módulos principales. } \\
\text { Baja funcionalidad }\end{array}$ & $\begin{array}{l}\text { Un DOF en } \\
\text { cada pata. }\end{array}$ & 5 motores \\
\hline $\begin{array}{l}\text { Salamandra } \\
\text { Robótica II [21] }\end{array}$ & $\begin{array}{l}\text { Posee } 9 \text { módulos distribuidos } \\
\text { a lo largo de la columna. } \\
\text { Alta funcionalidad }\end{array}$ & $\begin{array}{l}\text { Un DOF en } \\
\text { cada pata. }\end{array}$ & 12 motores \\
\hline Pleurobot [20] & $\begin{array}{l}\text { Posee } 12 \text { módulos en toda la } \\
\text { columna. Alta funcionalidad }\end{array}$ & $\begin{array}{l}\text { Cuatro DOF } \\
\text { en cada pata. }\end{array}$ & 19 motores \\
\hline Nuestro Enfoque & $\begin{array}{l}\text { Posee } 5 \text { módulos en la columna. } \\
\text { Funcionalidad media }\end{array}$ & $\begin{array}{l}\text { Un DOF en } \\
\text { cada pata. }\end{array}$ & 6 motores \\
\hline
\end{tabular}

En la Tabla 1 se observa la cantidad de módulos y motores utilizados en robots salamandra encontradas en la literatura. Nuestro prototipo presenta una complejidad media y utiliza un número reducido de elementos para su movimiento. El movimiento obtenido utilizando este diseño se ha comparado con el de una salamandra real; los resultados del desempeño del prototipo son presentados en la sección 3 .

\section{Resultados y discusión}

Para analizar y comparar el movimiento del robot con el movimiento de una salamandra real, se tomó como referencia un video en perspectiva cenital de la caminata de una salamandra real que se encuentra disponible en [26]. Utilizando morfología matemática se llevó a cabo la esqueletización de las imágenes obtenidas (Figura 8) y se extrajo el movimiento de la salamandra sin considerar sus extremidades.
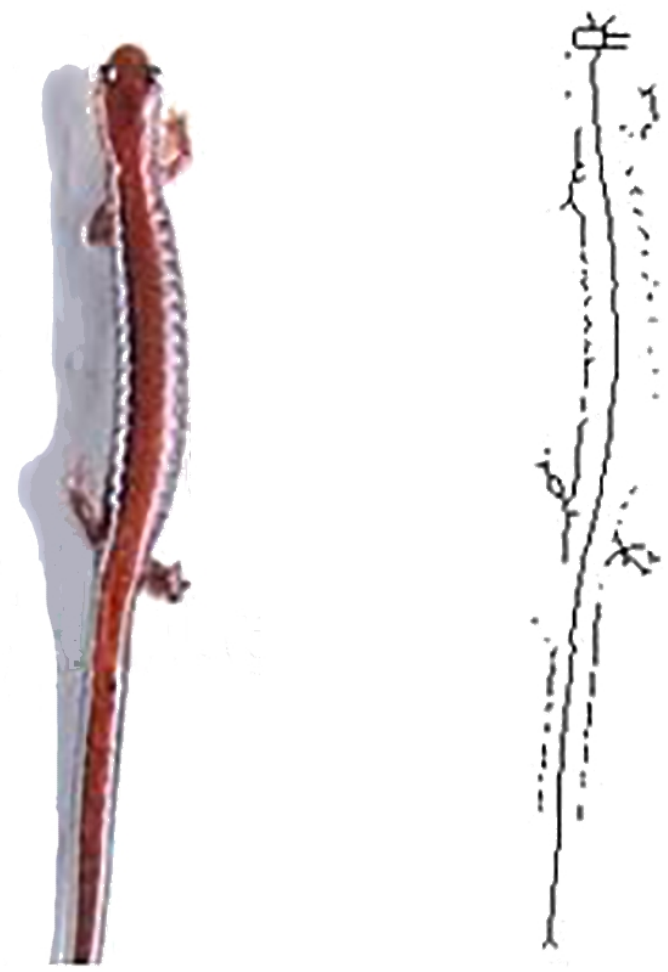

Figura 8. Esqueletización de la imagen para comparación de movimiento. (a) Imagen original (b) Imagen esqueletizada.

\subsection{Simulación}

Una vez adquiridos los datos del movimiento real del animal se realizó una simulación cinemática descomponiendo al robot en eslabones y articulaciones (Figura $9)$.

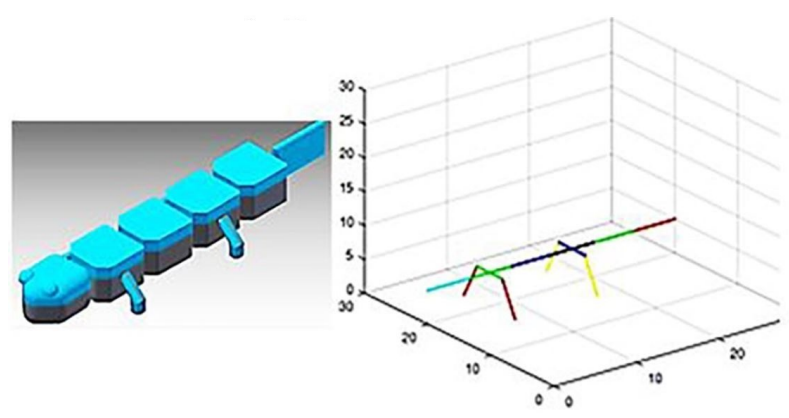

Figura 9. Descomposición en eslabones. 
Esta simulación se la realizó mediante geometría computacional [23] utilizando el diseño propuesto.

Una vez verificado el comportamiento cinemático del esqueleto de la salamandra se hizo una simulación CAD de la estructura completa.

\subsection{Acoplamiento de encoder}

Para la selección del acople del encoder se realizaron pruebas con dos tipos de transmisión: poleas y engranes. Se obtuvieron diferentes ángulos como respuesta al conteo de pulsos del encoder Los resultados se muestran en la Tabla 2. Y de forma gráfica en la Figura 10.

Tabla 2. Comparación del primer prototipo con robots presentados en la literatura

\begin{tabular}{ccccccc}
\hline \multicolumn{7}{c}{ Pulsos del encoder } \\
Ángulos & Engranes & \multicolumn{3}{c}{ Poleas } \\
& P1 & P2 & P3 & P1 & P2 & P3 \\
\hline $\mathbf{3 0}^{\mathbf{o}}$ & 1 & 1 & 1 & 6 & 4 & 3 \\
$\mathbf{6 0}^{\mathbf{o}}$ & 2 & 1 & 2 & 4 & 7 & 5 \\
$\mathbf{9 0}^{\mathbf{o}}$ & 3 & 2 & 2 & 9 & 11 & 9 \\
$\mathbf{1 2 0}^{\mathbf{o}}$ & 3 & 3 & 3 & 10 & 10 & 10 \\
$\mathbf{1 5 0}^{\mathbf{o}}$ & 4 & 4 & 4 & 13 & 12 & 12 \\
$\mathbf{1 8 0}^{\mathbf{o}}$ & 5 & 5 & 4 & 14 & 14 & 14 \\
$\mathbf{2 1 0}^{\mathbf{o}}$ & 6 & 6 & 6 & 16 & 16 & 16 \\
$\mathbf{2 4 0}^{\mathbf{o}}$ & 7 & 6 & 7 & 10 & 14 & 12 \\
$\mathbf{2 7 0}^{\mathbf{o}}$ & 8 & 7 & 7 & 11 & 17 & 17 \\
$\mathbf{3 0 0}^{\mathbf{o}}$ & 8 & 8 & 8 & 14 & 20 & 15 \\
$\mathbf{3 3 0}^{\mathbf{o}}$ & 9 & 3 & 9 & 17 & 19 & 17 \\
$\mathbf{3 6 0}^{\mathbf{o}}$ & 10 & 10 & 11 & 15 & 23 & 18 \\
\hline
\end{tabular}

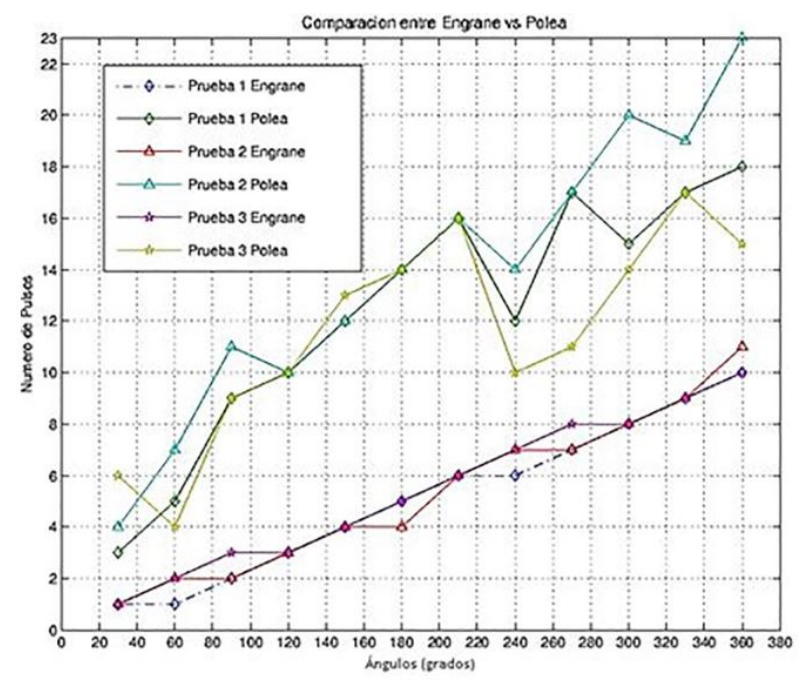

Figura 10. Descomposición en eslabones.

Para un adecuado funcionamiento del acople se requiere que la respuesta del encoder sea lineal. Como métrica de evaluación se tomó el coeficiente de determinación para la regresión lineal, cuya fórmula viene dada por la ecuación 1.

$$
R^{2}=\frac{\sigma_{X Y}^{2}}{\sigma_{X}^{2} \sigma_{Y}^{2}}
$$

Donde:

$\sigma_{\mathrm{XY}}$ Es la covarianza de $(\mathrm{X}, \mathrm{Y})$

$\sigma_{\mathrm{X}} \quad$ Es la desviación típica de la variable $\mathrm{X}$

$\sigma_{\mathrm{Y}} \quad$ Es la desviación típica de la variable $\mathrm{Y}$

El coeficiente de determinación es mejor mientras sea más cercano a 1. El promedio del coeficiente de determinación para las tres pruebas en engranes y poleas se muestra en la Tabla 3.

Tabla 3. Coeficientes de determinación promedio obtenidos en las pruebas

\begin{tabular}{cc}
\hline Coeficiente de determinación $\left(\mathbf{R}^{2}\right)$ \\
\hline Engranes & Poleas \\
0,882 & 0,801 \\
\hline
\end{tabular}

Basándose en los resultados obtenidos en la Tabla 3 se eligió la transmisión por engranes porque su $\mathrm{R}^{2}$ está más cerca del 1 en comparación con las poleas.

\subsection{Comparación de movimientos}

Se comparó los ángulos formados por la espina de la salamandra en el movimiento de derecha e izquierda al caminar (Figura 11). Los ángulos se midieron tomando como vértice el centro de la curvatura con un radio constante.

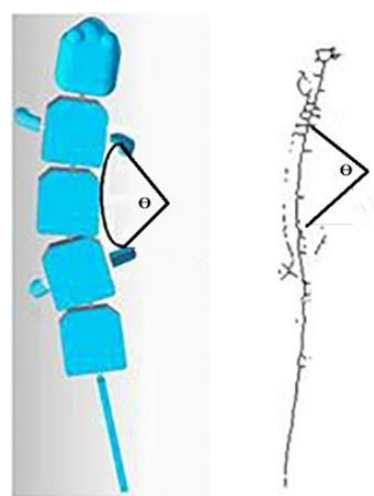

a)

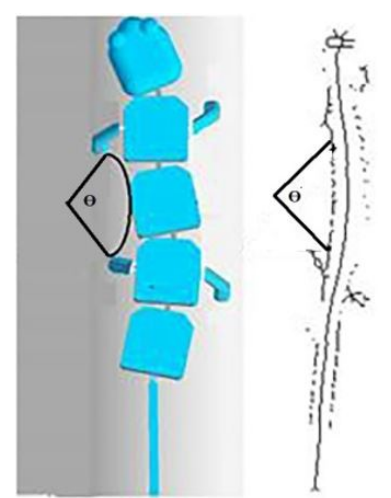

b)
Figura 11. Comparación de movimiento del robot con una salamandra real. (a) Ángulo flexión derecha. (b) Ángulo flexión izquierda.

Se hicieron diez pruebas con la extracción de tres ciclos de movimiento de la salamandra real y se comparó con tres ciclos de movimiento del robot y la simulación (cada ciclo se definió como dos pasos hacia adelante de 
la salamandra ya que se puede apreciar el movimiento de la columna hacia la izquierda y la derecha).

Los datos obtenidos de simulación (S), salamandra real $(\mathrm{R})$ y del prototipo $(\mathrm{P})$ se muestran en las Tablas 4,5 y 6 .

Tabla 4. Coeficientes de determinación promedio obtenidos en las pruebas

\begin{tabular}{ccccccc}
\hline \multicolumn{9}{c}{ Ángulos de giro (radianes) } \\
N.o & \multicolumn{3}{c}{ Derecha } & \multicolumn{3}{c}{ Izquierda } \\
& $\mathbf{R}$ & $\mathbf{S}$ & $\mathbf{P}$ & $\mathbf{R}$ & $\mathbf{S}$ & $\mathbf{P}$ \\
\hline $\mathbf{1}$ & 1,4 & 1,3 & 1,4 & 1,4 & 1,4 & 1,4 \\
$\mathbf{2}$ & 1,4 & 1,3 & 1,3 & 1,4 & 1,4 & 1,4 \\
$\mathbf{3}$ & 1,4 & 1,4 & 1,4 & 1,4 & 1,4 & 1,4 \\
$\mathbf{4}$ & 1,4 & 1,4 & 1,3 & 1,4 & 1,4 & 1,4 \\
$\mathbf{5}$ & 1,4 & 1,4 & 1,4 & 1,4 & 1,4 & 1,4 \\
$\mathbf{6}$ & 1,4 & 1,4 & 1,4 & 1,4 & 1,4 & 1,4 \\
$\mathbf{7}$ & 1,4 & 1,4 & 1,3 & 1,4 & 1,4 & 1,4 \\
$\mathbf{8}$ & 1,4 & 1,4 & 1,4 & 1,4 & 1,4 & 1,4 \\
$\mathbf{9}$ & 1,4 & 1,4 & 1,4 & 1,4 & 1,4 & 1,4 \\
$\mathbf{1 0}$ & 1,4 & 1,4 & 1,4 & 1,4 & 1,4 & 1,4 \\
\hline
\end{tabular}

Tabla 5. Coeficientes de determinación promedio obtenidos en las pruebas

\begin{tabular}{ccccccc}
\hline \multicolumn{8}{c}{ Ángulos de giro (radianes) } \\
N.o & \multicolumn{3}{c}{ Derecha } & \multicolumn{3}{c}{ Izquierda } \\
& $\mathbf{R}$ & $\mathbf{S}$ & $\mathbf{P}$ & $\mathbf{R}$ & $\mathbf{S}$ & $\mathbf{P}$ \\
\hline $\mathbf{1}$ & 1,4 & 1,4 & 1,4 & 1 & 1,4 & 1,4 \\
$\mathbf{2}$ & 1,4 & 1,4 & 1,4 & 1 & 1,4 & 1,4 \\
$\mathbf{3}$ & 1,4 & 1.41 & 1,4 & 1 & 1,4 & 1,4 \\
$\mathbf{4}$ & 1,4 & 1,4 & 1,3 & 1 & 1,4 & 1,4 \\
$\mathbf{5}$ & 1,4 & 1,4 & 1,5 & 1 & 1,4 & 1,4 \\
$\mathbf{6}$ & 1,4 & 1,4 & 1,4 & 1 & 1,4 & 1,4 \\
$\mathbf{7}$ & 1,4 & 1,4 & 1,4 & 1 & 1,4 & 1,4 \\
$\mathbf{8}$ & 1,4 & 1,4 & 1,4 & 1 & 1,4 & 1,4 \\
$\mathbf{9}$ & 1,4 & 1,4 & 1,4 & 1 & 1,4 & 1,4 \\
$\mathbf{1 0}$ & 1,4 & 1,4 & 1,4 & 1 & 1,4 & 1,4 \\
\hline
\end{tabular}

Tabla 6. Coeficientes de determinación promedio obtenidos en las pruebas

\begin{tabular}{ccccccc}
\hline \multicolumn{9}{c}{ Ángulos de giro (radianes) } \\
N.o & \multicolumn{3}{c}{ Derecha } & \multicolumn{3}{c}{ Izquierda } \\
& $\mathbf{R}$ & $\mathbf{S}$ & $\mathbf{P}$ & $\mathbf{R}$ & $\mathbf{S}$ & $\mathbf{P}$ \\
\hline $\mathbf{1}$ & 1,4 & 1,4 & 1,4 & 1,4 & 1,4 & 1,4 \\
$\mathbf{2}$ & 1,4 & 1,4 & 1,4 & 1,4 & 1,4 & 1,4 \\
$\mathbf{3}$ & 1,4 & 1,4 & 1,4 & 1,4 & 1,4 & 1,4 \\
$\mathbf{4}$ & 1,4 & 1,4 & 1,4 & 1,4 & 1,4 & 1,4 \\
$\mathbf{5}$ & 1,4 & 1,4 & 1,4 & 1,4 & 1,4 & 1,3 \\
$\mathbf{6}$ & 1,4 & 1,4 & 1,4 & 1,4 & 1,5 & 1,4 \\
$\mathbf{7}$ & 1,4 & 1,4 & 1,4 & 1,4 & 1,4 & 1,4 \\
$\mathbf{8}$ & 1,4 & 1,4 & 1,4 & 1,4 & 1,4 & 1,4 \\
$\mathbf{9}$ & 1,4 & 1,4 & 1,4 & 1,4 & 1,4 & 1,4 \\
$\mathbf{1 0}$ & 1,4 & 1,4 & 1,4 & 1,4 & 1,4 & 1,3 \\
\hline
\end{tabular}

En las Figuras 12, 13 y 14 se puede apreciar la variación en los ángulos para los tres ciclos de movimiento de acuerdo con los datos tabulados anteriormente.

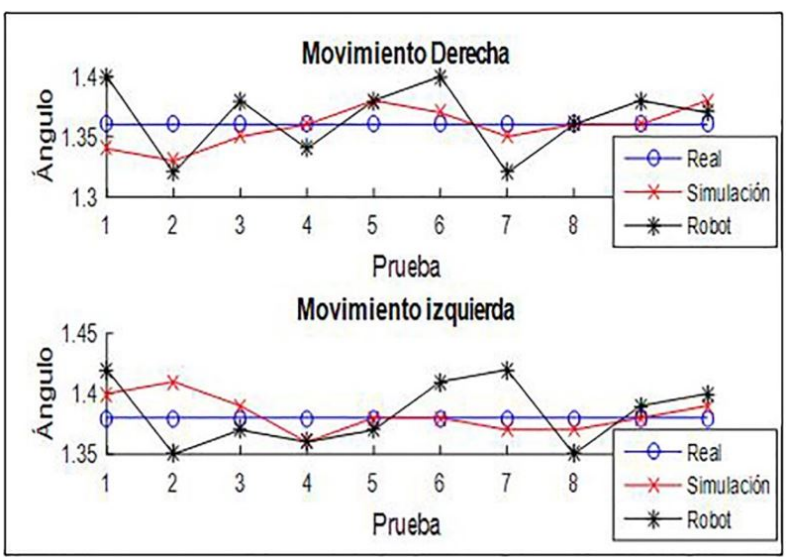

Figura 12. Variación de ángulos durante el primer ciclo de movimiento.

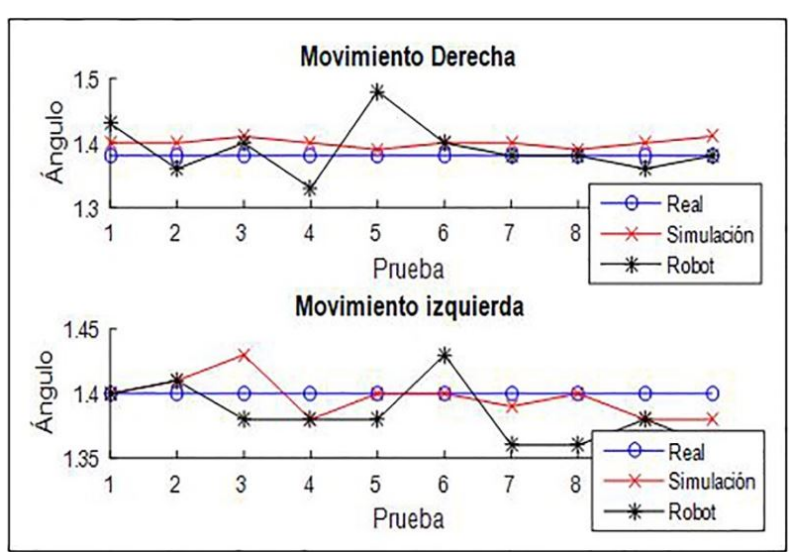

Figura 13. Variación de ángulos durante el segundo ciclo de movimiento.

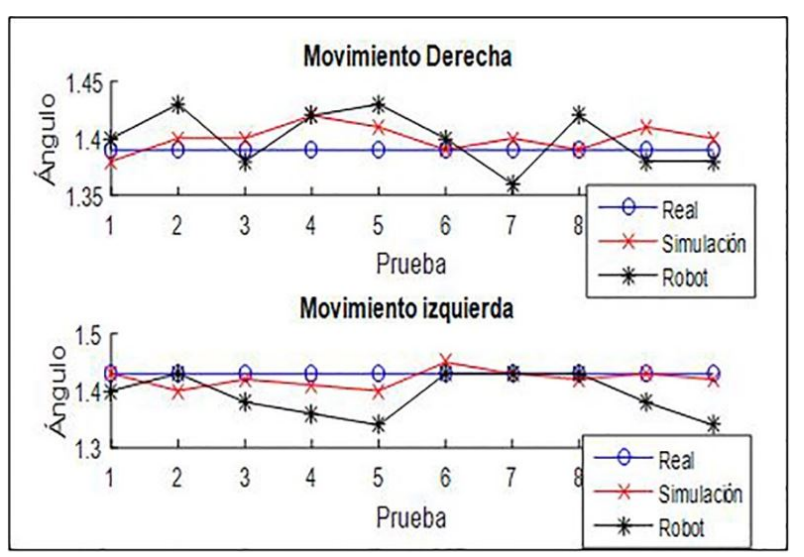

Figura 14. Variación de ángulos durante el tercer ciclo de movimiento.

Para determinar la precisión de los ángulos de movimiento se utilizó como métrica de evaluación el error cuadrático medio $[24,25]$, dado por la ecuación 2: 


$$
R M S E=\sqrt{\frac{1}{n} \sum_{t=1}^{n}\left(Y_{t}-\hat{Y}_{t}\right)^{2}}
$$

En la Tabla 7 se muestran los resultados del RMSE obtenido para los diferentes ciclos de movimiento, se tabulan los errores obtenidos de la simulación y del robot con respecto al movimiento real de la salamandra.

Tabla 7. Cálculo del error cuadrático medio para los diferentes ciclos de movimiento

\begin{tabular}{cccc}
\hline Ciclo & Movimiento & Sim./Robot & RMSE \\
\hline \multirow{4}{*}{1} & \multirow{2}{*}{ Derecha } & Simulación & 0,015 \\
& & Robot & 0,028 \\
& Izquierda & Simulación & 0,014 \\
& & Robot & 0,026 \\
\multirow{2}{*}{2} & Derecha & Simulación & 0,021 \\
& & Robot & 0,041 \\
& Izquierda & Simulación & 0,015 \\
& & Robot & 0,027 \\
3 & \multirow{2}{*}{ Derecha } & Simulación & 0,015 \\
& \multirow{2}{*}{ Izquierda } & Robot & 0,026 \\
& & Simulación & 0,015 \\
& & Robot & 0,052 \\
\hline
\end{tabular}

Como se puede observar en la Tabla 7, el error máximo obtenido en la simulación es de 0,021, que representa aproximadamente el 1,5\% de ángulo de error con respecto al movimiento real de la salamandra, mientras que en el caso del prototipo, el error máximo obtenido fue de 0,052 , lo cual representa el $3,5 \%$ de error aproximadamente. El error medio obtenido en simulaciones y durante el movimiento del robot puede ser observado en la Tabla 8.

Tabla 8. Cálculo del error medio en todos los ciclos de movimiento

\begin{tabular}{cc}
\hline \multicolumn{2}{c}{ Error medio (\%) } \\
\hline Simulación & Robot \\
1,14 & $2,40 \%$ \\
\hline
\end{tabular}

Con base en estos datos podemos decir que la implementación de nuestro diseño imita el movimiento de la salamandra con un error en los ángulos de 2,4 \%

\section{Conclusiones}

La estructura de la salamandra adoptada al robot se traduce en un diseño sencillo para lograr que el prototipo propuesto como robot anfibio, tenga un buen desempeño en agua y en tierra.

El enfoque modular propuesto hace al prototipo versátil por utilizar dos diferentes tipos de elementos: el cuerpo y las extremidades (patas), que facilitan la alimentación de los circuitos y hace que los módulos se adapten para construir distintos tipos de robots.

La transmisión circular-circular entre engranes proporciona un censado de ángulo de movimientos casi lineal al usar un encoder rotativo.

En el prototipo se evidenció la importancia del movimiento de la columna, en la caminata dio un empuje a las patas ayudando en la movilización y en la natación proporcionó velocidad al reproducir el movimiento.

La comparación de movimientos demostró la similitud entre los movimientos al caminar de una salamandra real con el prototipo, siendo la diferencia menor al $5 \%$.

El bajo presupuesto se tradujo en un diseño simple, con una aceptable funcionalidad; una mayor inversión mejoraría algunos aspectos, debido al conocimiento que ha proporcionado el estudio y el primer prototipo.

\section{Agradecimientos}

El presente trabajo es parte del proyecto de investigación 2016 «Vehículo terrestre multipropósito no tripulado (MultiNavCar)» de la Universidad de las Fuerzas Armadas ESPE, dirigido por el Dr. Wilbert G. Aguilar.

\section{Referencias}

[1] R. Breithaupt, J. Dahnke, K. Zahedi, J. Hertzberg, and F. Pasemann, "Robo-Salamander - an approach for the benefit of both robotics and biology ," Proc. Fifth Int. Conf. Climbing Walk. Robot. their Support. Technol. CLAWAR 2002, 25-27th Sept. 2002, no. January, p. 55, 2002.

[2] W. G. Aguilar and C. Angulo, "Compensación de los Efectos Generados en la Imagen por el Control de Navegación del Robot Aibo ERS 7," Memorias del VII Congr. Cienc. y Tecnol. ESPE 2012, pp. 165-170, 2012.

[3] —, "Compensación y Aprendizaje de Efectos Generados en la Imagen durante el Desplazamiento de un Robot," X Simposio CEA de Ingeniería de Control, 2012.

[4] _ _ , "Robust video stabilization based on motion intention for low-cost micro aerial vehicles," MultiConference on Systems, Signals Devices (SSD), 2014 11th International, pp. 1-6, 2014.

[5] —_ "Estabilización de video en micro vehículos aéreos y su aplicación en la detección de caras," Memorias del IX Congr. Cienc. y Tecnol. ESPE 2014, pp. 155-160, 2011. 
[6] R. Pfeifer, M. Lungarella, and F. Iida, "Self- Organization, Embodiment, and Biologically Inspired Robotics," Science, vol. 318, no. 2007, pp. 10881093, 2007.

[7] A. J. Ijspeert, A. Crespi, and J.-M. Cabelguen, "Simulation and robotics studies of salamander locomotion: applying neurobiological principles to the control of locomotion in robots," Neuroinformatics, vol. 3, no. 3, pp. 171-195, 2005.

[8] D. R. Carrier, "Action of the Hypaxial Muscles During Walking and Swimming in the Salamander Dicamptodon Ensatus," J. exp. Biol, vol. 180, pp. 75-83, 1993.

[9] M. A. Ashley-Ross and G. V. Lauder, "Motor patterns and kinematics during backward walking in the pacific giant salamander: evidence for novel motor output." Journal of neurophysiology, vol. 78, pp. 3047-3060, 1997.

[10] A. Crespi, "Design and Control of Amphibious Robots with Multiple Degrees of Freedom," Optimization, vol. 3786, 2007.

[11] J. Monsalve, J. León, and K. Melo, "Modular snake robot oriented open simulation software," 4 th Annual IEEE International Conference on Cyber Technology in Automation, Control and Intelligent Systems, IEEE-CYBER 2014, pp. 546-550, 2014.

[12] P. Liljebäck, S. Fjerdingen, K. Y. Pettersen, and Ø. Stavdahl, "A snake robot joint mechanism with a contact force measurement system," Proceedings - IEEE International Conference on Robotics and Automation, no. 978, pp. 3815-3820, 2009.

[13] N. Kamamichi, M. Yamakita, K. Asaka, and Z. W. Luo, "A snake-like swimming robot using IPMC actuator/sensor," Proceedings - IEEE International Conference on Robotics and Automation, vol. 2006, no. May, pp. 1812-1817, 2006.

[14] S. Yu, S. Ma, B. Li, and Y. Wang, "An amphibious snake-like robot with terrestrial and aquatic gaits," Proceedings - IEEE International Conference on Robotics and Automation, pp. 2960-2961, 2011.

[15] K. Hirata, "Development of Experimental Fish Robot," Sixth International Symposium On Marine Engineering, no. 650, pp. 711-714, 2000.

[16] F. W. Grasso, T. R. Consi, D. C. Mountain, and J. Atema, "Biomimetic robot lobster performs chemo-orientation in turbulence using a pair of spatially separated sensors: Progress and challenges," Robotics and Autonomous Systems, vol. 30, no. 1, pp. 115-131, 2000.

[17] U. Saranli, M. Buehler, and D. E. Koditschek, "RHex: A Simple and Highly Mobile Hexapod Robot," The International Journal of Robotics Research, vol. 20, no. July, pp. 616-631, 2001.

[18] A. S. Boxerbaum, P. Werk, R. D. Quinn, and R. Vaidyanathan, "Design of an autonomous amphibious robot for surf zone operation: part I mechanical design for multi-mode mobility," Proceedings, 2005 IEEE/ASME International Conference on Advanced Intelligent Mechatronics, pp. 1459-64, 2005.

[19] S. Guo, S. Mao, L. Shi, and M. Li, "Design and kinematic analysis of an amphibious spherical robot," 2012 IEEE International Conference on Mechatronics and Automation, ICMA 2012, pp. 2214-2219, 2012.

[20] T. Horvat, "Control of a Salamander-Like Robot for Search and Rescue Applications," pp. 1-8.

[21] A. Crespi, K. Karakasiliotis, A. Guignard, and A. J. Ijspeert, "Salamandra Robotica II: An amphibious robot to study salamander-like swimming and walking gaits," IEEE Transactions on Robotics, vol. 29, no. 2, pp. 308-320, 2013.

[22] A. Crespi and A. J. Ijspeert, "Salamandra Robotica: A biologically inspired amphibious robot that swims and walks," Artificial Life Models in Hardware, pp. 35-64, 2009.

[23] W. G. Aguilar and S. Morales, "3D Environment Mapping Using the Kinect V2 and Path Planning Based on RRT Algorithms," Electronics, vol. 5, no. 4, p. 70, 2016.

[24] W. G. Aguilar and C. Angulo, "Real-Time ModelBased Video Stabilization for Microaerial Vehicles," Neural Process. Lett., vol. 43, no. 2, pp. 459-477, 2016.

[25] —, "Real-time video stabilization without phantom movements for micro aerial vehicles," EURASIP J. Image Video Process, no. 1, p. 46, 2014.

[26] Shutterstock. Salamander walks across white surface, shot from above. Vibe Images. [Online]. Available: http://www.shutterstock.com/es/video/ clip-8807212-stock-footage-salamander-walks- $\backslash$ across-white-surface-shot-from-above.html? src= $\mathrm{rel} / 8807005: 1 / \mathrm{gg}$. 